\title{
O EXPERIMENTO DE CLEMENT-DESORMES NO SÉCULO XXI
}

\author{
Otávio Luiz Bottecchia* \\ Instituto de Química, Universidade Federal de Uberlândia, Av. João Naves de Ávila, 2121, 38408-902 Uberlândia - MG, Brasil
}

Recebido em 20/8/09; aceito em 6/4/10; publicado na web em 30/7/10

\begin{abstract}
THE CLEMENT-DESORMES EXPERIMENT IN THE $21^{\text {st }}$ CENTURY. The Clement-Desormes experiment is reviewed. By reason of a finite difference between the pressure within the system and its surroundings, Bertrand and McDonald have criticized the usual consideration of the adiabatic expansion as reversible. Garland, Nibler and Shoemaker oppose, defining regions through virtual boundaries where the surroundings do not operate. For Holden, the use of virtual boundaries is expendable. Experiments cannot support a hypothesis testing due to experiment's intrinsic uncertainty. The role of polytropy in uncertainty is discussed. Both thermodynamic definitions and kinetic model depict the real processes as irreversible phenomena and the reversible ones as a limiting hypothetical case.
\end{abstract}

Keywords: heat capacity; reversible; Clement-Desormes.

\section{INTRODUÇÃO}

O experimento de Clement-Desormes ${ }^{1}$ data de 1819. Aperfeiçoado posteriormente por Gay-Lussac e Welter, ${ }^{2}$ objetiva determinar a relação, $\gamma$, entre as capacidades caloríficas molares de um gás à pressão constante, $C_{P, m}$, e a volume constante, $C_{V, m}: \gamma=C_{P, m} / C_{V, m}$. Mais precisamente, objetiva determinar $C_{V, m}$, já que, para alguns autores, ${ }^{3} \gamma$ é uma relação de conveniência dependente da validade de $C_{P, m}-C_{V, m}=R$.

Neste experimento, o escape do gás tem sido descrito no contexto de um sistema fechado. Introduziram-se fronteiras imaginárias e deduziram-se equações que — argumenta-se —, se aplicam apenas à parte do gás que permanece no recipiente após a rolha ter sido recolocada. ${ }^{4}$ Revelaram-se empecilhos para, por exemplo, explicar como uma equação faria distinção entre as partes de um sistema homogêneo, fechado e em equilíbrio; ou, ainda, explicar qual seria o critério para determinar o limite da fronteira imaginária. Holden ${ }^{5}$ prescindiu destes artifícios e formulou o escape em sistema aberto.

A reversibilidade foi questionada por Bertrand e McDonald, ${ }^{6}$ porque a expansão do gás ocorre contra a atmosfera, cuja oposição é constante. A reversibilidade exige pressão opositora variável. Por isso, eles apresentaram uma nova proposição. A politropia (troca de calor entre um sistema termodinâmico e sua vizinhança favorecida pela imperfeição do isolamento térmico) é quase um corolário da imposição da reversibilidade, a qual exige lentidão. Em contrapartida, lentidão favorece troca de calor, que, no presente caso, é fonte de erro.

Os aspectos que fazem do experimento de Clement-Desormes objeto de revisão são: o escape do gás; a reversibilidade da expansão de um gás contra a atmosfera; a politropia. Trata-se de tema acadêmico, no escopo da Físico-química.

\section{DESCRIÇÃO DO EXPERIMENTO}

Enche-se um recipiente grande $(20 \mathrm{~L})$ de parede grossa $(6 \mathrm{~mm})$ com um gás numa pressão, $P_{1}$, maior que a pressão atmosférica, $P_{a t m}$. Aguarda-se o equilíbrio térmico. Abre-se e fecha-se rapidamente $(1 \mathrm{~s})$ o recipiente, de modo que as pressões interna e atmosférica se igualem, $P_{2}=P_{a t m}$. O gás se resfria, pois a parede grossa, além de um gargalo largo $(50 \mathrm{~mm})$, minimiza a troca de calor e o tempo entre o abrir e o fechar. $\mathrm{O}$ final desta expansão encerra o primeiro processo e inicia o segundo. Incontinenti, monitora-se a pressão interna, que aumenta paulatinamente. O aumento isocórico (volume constante) da pressão no sistema fechado demonstra que a vizinhança está mais quente que o gás. Após equilibrar-se, a pressão do recipiente é $P_{3}$. A Tabela 1 resume os estados do gás e define os processos " 1 " e " 2 ".

Tabela 1. Descrição dos estados do gás no recipiente ao longo do experimento de Clement-Desormes. $T_{a m b}$ representa a temperatura da vizinhança. A quantidade do gás, $n$, e o volume, $V$, em qualquer dos estados, não costumam ser determinados experimentalmente. $\mathrm{O}$ processo 1 é considerado adiabático. $\mathrm{O}$ processo 2 é isocórico, cuja irreversibilidade é irrelevante para as questões tratadas neste trabalho. Admitindo-se comportamento ideal, é válida a equação $\frac{P_{i} V_{i}}{n_{i} T_{i}}=\frac{P_{j} V_{j}}{n_{j} T_{j}}, i, j=1,2 \mathrm{e} 3$, com ou sem escape

\begin{tabular}{lccc}
\hline & $\begin{array}{c}\text { Início do } \\
\text { processo 1 }\end{array}$ & $\begin{array}{c}\text { Fim do processo 1 } \\
\text { Início do processo 2 }\end{array}$ & $\begin{array}{c}\text { Fim do } \\
\text { processo 2 }\end{array}$ \\
\hline Variável & Estado 1 & Estado 2 & Estado 3 \\
Pressão & $P_{1}>P_{A T M}$ & $P_{2}=P_{a t m}$ & $P_{3}>P_{2}$ \\
Volume & $V_{1}$ & $V_{2}=V_{1}$ & $V_{3}=V_{2}=V_{1}$ \\
Temperatura & $T_{1}=T_{a m b}$ & $T_{2}<T_{1}$ & $T_{3}=T_{1}=T_{a m b}$ \\
Quantidade & $n_{1}$ & $n_{2}<n_{1}{ }^{(1)}$ & $n_{3}=n_{2}$ \\
Volume & $V_{1, \mathrm{~m}}{ }^{(1)}$ & $V_{2, m}>V_{1, m}$ & $V_{3, m}=V_{2, m}$ \\
molar $^{(2)}$ & & &
\end{tabular}

(1) Garland, Nibler e Shoemaker ${ }^{4}$ conceberam o experimento com volume menor que o volume geométrico do recipiente; quando o recipiente é aberto, segundo eles, o sistema se expande contra o gás acima até ocupar o volume geométrico do recipiente. (2) $\mathrm{O}$ volume molar do gás no estado $i$, $V_{i, m}$, é definido por $V_{i, m}=\frac{V_{i}}{n_{i}}, i=1,2$ ou 3 .

Medem-se $P_{1}$ e $P_{3}$ com um barômetro sensível. Consegue-se sensibilidade de $10 \mathrm{~Pa}$ usando água num manômetro diferencial de extremidade aberta para a atmosfera e fixado sobre uma régua, cuja menor divisão seja $1 \mathrm{~mm}$. Maior sensibilidade consegue-se usando ftalato de dibutila. ${ }^{7}$ Mede-se $P_{a t m}$ com um barômetro de mercúrio com extremidade fechada para a atmosfera (ou com um barômetro comercial). A relação entre as capacidades caloríficas determina-se pela Equação 1 ou pela Equação

$$
\frac{C_{P, m}}{C_{V, m}}=\frac{\ln \left(P_{1} / P_{2}\right)}{\ln \left(P_{1} / P_{3}\right)}
$$

(1, reversível) 


$$
\frac{C_{P, m}}{C_{V, m}}=\frac{\left(P_{1} / P_{2}\right)-1}{\left(P_{1} / P_{3}\right)-1}
$$

A diferença conceitual entre a Equação 1 e a 2 está, respectivamente, na suposição da reversibilidade ou irreversibilidade do processo 1 . O sucesso do experimento deve-se, em parte, à possibilidade de calcular $\gamma$ apenas a partir das pressões, $P_{1}, P_{2}$ e $P_{3}$. Se o resultado do experimento dependesse de medição da temperatura, a capacidade calorífica (não nula) do termômetro, aliada ao seu tempo de resposta, frustraria a simplicidade do experimento. Por isso, os dois tratamentos descritos a seguir buscam combinar as relações matemáticas para isolar a pressão das demais variáveis.

\section{TRATAMENTO COMO SISTEMA FECHADO}

\section{Hipótese: Processo 1 é reversível}

Admite-se $\mathrm{s}^{4}$ comportamento ideal e validade da Equação 3:

$$
C_{P, m}-C_{V, m}=R
$$

Genericamente, ${ }^{8} C_{p}-C_{v}=\alpha^{2} T V / \kappa_{T}$, em que $\alpha$ é o coeficiente de expansão e $\kappa_{T}$ é o coeficiente de compressibilidade isotérmica do gás, (a ressalva no primeiro parágrafo da Introdução relaciona-se a esta equação). A reversibilidade do processo 1 requer $\ln P_{2} / P_{1}=-\gamma \ln V_{2, m} / V_{1, m}$. No processo 2, isocórico e sem escape de gás, vale a Equação 4. Combinando estas equações, obtém-se a Equação 1.

$$
P_{3} / T_{1}=P_{2} / T_{2}
$$

Admitiu-se que o gás, inicialmente no recipiente, seja dividido em duas partes por uma superfície imaginária; a parte acima da superfície deixa o recipiente quando a rolha é removida e, presumivelmente, interage irreversivelmente com a vizinhança, mas a parte abaixo da superfície expande-se reversivelmente contra a superfície imaginária. ${ }^{4}$ Esta concepção traz duas objeções: a primeira, sobre a reversibilidade, discutida na seção Caracterização; a segunda, sobre o volume do recipiente no final do primeiro processo. O recipiente é rígido, portanto, seu volume geométrico não varia. Assumir que $V_{2}=V_{1}$ significa assumir que não há expansão e, neste caso, tratar o sistema como fechado significa assumir que $V_{2, m}=V_{1, m}$. Daí a necessidade de uma superfície imaginária. Para concretizar tal superfície, Buep, Czekalski e Baróm ${ }^{9}$ modificaram a concepção experimental introduzindo um cilindro munido de um pistão móvel conectado ao recipiente e uma válvula substituindo a rolha. Além das condições da Tabela 1, há então o volume do cilindro, inicialmente zero. Abre-se a válvula e o gás expande-se do recipiente para o cilindro. O volume no cilindro após a expansão é diferente dependendo da hipótese de reversibilidade ou irreversibilidade. A formulação desenvolve-se com base neste volume que, eliminado, leva à Equação 1.

\section{Hipótese: Processo 1 é irreversível}

Bertrand e McDonald ${ }^{6}$ consideram o gás expandindo-se contra uma pressão opositora constante e igual à atmosférica: $n C_{V, m} d T=-P_{2} d V$. - Bertrand ${ }^{10}$ detectou nos livros didáticos a formulação desta equação conforme quatro protocolos. Isto traz obstáculo desnecessário à compreensão do experimento. A definição de trabalho proposta por Beattie ${ }^{11}$ unifica esses protocolos. - Por integração: $n C_{V, m}\left(T_{2}-T_{1}\right)=-P_{2}\left(\frac{n R T_{2}}{P_{2}}-\frac{n R T_{1}}{P_{1}}\right)$, em que $n$ se refere à quantidade idealizada no experimento; tacitamente, é considerada constante. Esta equação, a Equação 3 e a 4 levam à Equação 2.
Nesta formulação, o processo 2 se inicia com o aprisionamento do gás no volume $V_{1}$, na temperatura $T_{2}$ e pressão $P_{2}=P_{a t m}$, e transcorre isocoricamente até a temperatura voltar a $T_{1}$. A nova pressão no final deste processo seria $P_{3}$. Esta idealização mascara o escape de gás. Do contrário, depreende-se que $V_{2}$ não é mais o volume geométrico do recipiente. A contradição surge na aplicação da Equação 4, que pressupõe $V_{2}=V_{1}$ e $n_{2}=n_{1}$. Por isto, para justificar a Equação 2, é necessário, novamente, lançar mão de uma superfície imaginária.

\section{TRATAMENTO COMO SISTEMA ABERTO}

Deve-se a Holden ${ }^{5}$ a solução dos problemas na definição da fronteira e do escape de gás. O experimento de Clement-Desormes seria um exemplo provocador de um experimento em que o sistema aberto é tratado como sistema fechado. A expressão diferencial do balanço energético de um elemento de volume em um sistema contínuo, com um componente, monofásico, realizando trabalho $w$ na ausência de campos é: ${ }^{12}$

$$
d U=d q+d w+H_{m} d n
$$

onde, $U$ é a energia interna do gás; $q$ é o calor trocado e $H_{m}$ é a entalpia molar.

\section{Hipótese: Processo 1 é reversível}

Holden caracteriza a reversibilidade igualando as entalpias molares dentro e fora do recipiente. Admite-se que: o volume seja constante $(d w=0)$ e o processo 1 seja adiabático $(d q=0)$. A diferencial total da energia interna é $d U=d\left(n U_{m}\right)=n d U_{m}+U_{m} d n$. A Equação 5 tornase $n d U_{m}=\left(H_{m}-U_{m}\right) d n$. Com $d U_{m}=C_{V, m}^{m} d T$ e com a entalpia do gás ideal, $H_{m}=U_{m}+R T$, obtém-se:

$$
\frac{C_{V, m} d T}{R T}=\frac{d n}{n}
$$

A partir da Equação 6 integrada, desde que válida a Equação 3, obtém-se a Equação 1.

\section{Hipótese: Processo 1 é irreversível}

Holden caracteriza a irreversibilidade através da descontinuidade das propriedades intensivas no jato de gás e no recipiente. Internamente, a entalpia molar do gás varia no tempo; externamente, permanece constante. Assim, $\hat{H}_{m}$ não depende de $\hat{n}$. O símbolo "^" diferencia uma propriedade no jato de gás da mesma propriedade no recipiente. Admite-se, junto com as demais suposiccões do caso anterior, que a expansão seja isoentálpica, $H_{m} d n+\hat{H}_{m} d \hat{n}=0$. A Equação 5 torna-se $d\left(n U_{m}\right)=\hat{H}_{m} d n$, porque o balanço de massa impõe $d n=-d \hat{n}$. Após integração e rearranjo: $n_{2}\left(U_{m, 2}-\hat{U}_{m}-R \hat{T}\right)=n_{1}\left(U_{m, 1}-\hat{U}_{m}-R \hat{T}\right)$. A diferença de energia interna dentro dos parênteses relaciona-se com a capacidade calorífica: $\left(U_{m}-\hat{U}_{m}\right)=C_{V, m}(T-\hat{T})$. O uso da Equação 3 fornece:

$$
C_{V \cdot m}\left(n_{2} T_{2}-n_{1} T_{1}\right)=C_{P . m}\left(n_{2} \hat{T}-n_{1} \hat{T}\right)
$$

Holden argumenta que, se todo o gás do escape fosse aprisionado em um frasco do mesmo tamanho do recipiente, na pressão atmosférica, $P_{2}=P_{\text {atm }}$, então, ao ser aquecido isocoricamente, atingiria a pressão final $P_{3}: \hat{T}=P_{2} T_{1} / P_{3}$. Substituindo na Equação 7 e assumindo idealidade entre os estados 1 e 3 (Tabela 1) obtém-se a Equação 2. 


\section{CARACTERIZAÇÃO}

Bertrand e McDonald ${ }^{6}$ afirmam que o processo 1 é irreversível porque a expansão do gás ocorreria contra a atmosfera, que exerce pressão opositora constante. A reversibilidade exigiria pressão opositora igual à pressão do gás, que varia. Portanto, a reversibilidade não se aplicaria ao experimento. Garland, Nibler e Shoemaker discordam. ${ }^{4}$ A irreversibilidade traria a questão de onde a fronteira do sistema deve ser definida, visto que a pressão externa, $P_{2}\left[P_{2}=P_{a t m}\right]$, é exercida apenas fora [grifo deles] do gargalo do recipiente e visto que o tratamento requer um sistema fechado com pressão interna e temperatura uniformes. Holden ${ }^{5}$ mostra a resolução do problema nas hipóteses de reversibilidade e de irreversibilidade, mas furtou-se de caracterizar o processo 1. Para fazê-lo, revisa-se a seguir o conceito de reversibilidade.

\section{Concepção macroscópica}

Uma transformação é reversível quando cada estado sucessivo da transformação difere um infinitésimo do estado de equilíbrio. ${ }^{13}$ As transformações reversíveis são uma estilização da realidade; são transformações limites em direção às quais certas transformações reais podem tender quando colocadas em condições apropriadas. Não existe a todo rigor, transformação reversível na natureza. Todas as transformações são irreversíveis. ${ }^{14}$ Acrescenta-se a necessidade do estado das vizinhanças também ter de ser restaurado quando um sistema é modificado e restaurado ao seu estado inicial. ${ }^{15}$ Quando isto acontece, os estados intermediários nos processos de modificação e de restauração são os mesmos. De fato, reversibilidade é consequência da ausência de processos dissipativos. O oposto, a irreversibilidade termodinâmica, não significa impossibilidade de restaurar um sistema ao seu estado inicial. ${ }^{16}$

A definição de reversibilidade por uma diferença infinitesimal (ou infinitésimo) simplifica a definição alternativa: "uma transformação é reversível quando os estados sucessivos da transformação são estados de equilíbrio". Poder-se-ia ter a falsa impressão de que tal sistema não progride nem em direção ao estado final nem ao estado inicial, i. e., de que seria um sistema estático. A falsa impressão origina-se no hábito de afirmar que diferencial é o mesmo que uma diferença pequena. Bassi et al. ${ }^{17}$ alertaram sobre este problema em mais de uma ocasião. Diferencial não é uma diferença pequena; diferencial representa um ponto. Assim como, em Matemática, a diferença entre duas grandezas, no limite, representa um ponto, em Termodinâmica, a diferença entre dois estágios de um processo, no limite, representa um estado.

$\mathrm{Se}$, portanto, os processos reversíveis não são processos reais e os processos reais são sempre irreversíveis, ${ }^{15}$ pode-se dar o problema como resolvido completando o silogismo: o processo 1 é real; logo, é irreversível. Os argumentos contrários são refutados a seguir, onde afloram os empecilhos mencionados na introdução. Da discussão vem a sugestão de investigar a origem do erro das medidas. A concepção microscópica, além de complementar a presente seção, aponta na mesma direção.

\section{Argumentos de Garland, Nibler e Shoemaker}

O primeiro argumento contrário é a irreversibilidade trazer a questão de onde a fronteira do sistema deve ser definida, visto que a pressão externa é exercida apenas fora do gargalo do recipiente $e$ visto que o tratamento requer um sistema fechado com pressão interna e temperatura uniformes. Depreende-se que, se a atmosfera atuasse no gargalo, haveria uma descontinuidade. Descontinuidade caracteriza irreversibilidade. Em segundo lugar, a atmosfera já atua no gargalo antes da abertura da rolha. Inexiste uma força impelindo a atmosfera a atuar apenas fora do gargalo.
O segundo argumento faz uso do fato do numerador e do denominador da Equação 1 poderem ser escritos na forma de série infinita (Equação 8). As Equações 1 e 2 seriam iguais dentro do erro experimental, porque a Equação 2 é a Equação 8 truncada, com diferença de $2 \%$.

$$
\begin{gathered}
\left.\frac{\ln \left(P_{1} / P_{2}\right)}{\ln \left(P_{1} / P_{3}\right)}=\frac{\left(P_{1} / P_{2}-1\right)\left[1-(1 / 2)\left(P_{1} / P_{2}-1\right)+(1 / 3)\left(P_{1} / P_{2}-1\right)^{2}-\ldots\right]}{\left(P_{1} / P_{3}-1\right)\left[1-(1 / 2)\left(P_{1} / P_{3}-1\right)+(1 / 3)\left(P_{1} / P_{3}-1\right)^{2}-\ldots\right]}\right] \\
\frac{\ln \left(P_{1} / P_{2}\right)}{\ln \left(P_{1} / P_{3}\right)} \approx \frac{\left(P_{1} / P_{2}-1\right)}{\left(P_{1} / P_{3}-1\right)}
\end{gathered}
$$

Determinada a precisão de $\gamma$, é legítimo truncar a série infinita. Precisão não caracteriza reversibilidade ou irreversibilidade. A proximidade dos resultados das Equações 1 e 2 apenas demonstra que: o primeiro termo da série infinita é suficiente para descrever $\gamma$ com precisão compatível com os $2 \%$; a pressão do recipiente não é elevada o suficiente para que os termos entre colchetes da Equação 8 sejam significativos; o equipamento foi bem dimensionado, no caso da incerteza poder ser considerada pequena (a seção Suporte experimental mostra que o erro chega a mais de $8 \%$ ). Outros detalhes podem ser encontrados na literatura. ${ }^{9}$

A argumentação segue com três predições. Duas delas estão de acordo com Magarvey et al. ${ }^{18}$ e com a descrição do apêndice, sendo dispensável comentá-las aqui. Na outra, entretanto, consta uma indefinição: $\gamma$ seria relativamente baixo se a expansão apresentasse apreciável grau de irreversibilidade. Pelas definições acima, não há graus de reversibilidade: os processos ou são reversíveis ou irreversíveis. O último argumento vem na mesma linha argumentativa: não haveria irreversibilidade significativa se, durante a expansão, não houvesse gradientes de pressão significativos abaixo da superfície imaginária. Depara-se com conceito subjetivo: o que se entende por reversibilidade significativa? Uma possível resposta encontra-se na ponderação de Castellan: ${ }^{15}$ com paciência e sagacidade, podemos fazer um processo aproximar-se da reversibilidade, mas nunca atingi-la. Garland, Nibler e Shoemaker buscam as condições experimentais fazendo o processo irreversível aproximar-se do reversível, dentro do erro experimental. A otimização dos fatores experimentais torna plausível o tratamento do processo 1 como reversível, não enquanto fato, mas enquanto caso-limite.

\section{Concepção microscópica}

Bertrand ${ }^{10}$ revisou um debate ${ }^{19}$ entre Bauman, Chesik e Kokes sobre o trabalho de expansão/compressão isotérmico. Bauman teria sugerido que o trabalho realizado por um gás sobre um êmbolo fosse calculado através da pressão operante: $P_{\text {operante }}=P_{\text {gas }}\left(1 \pm \frac{u}{v_{x}}\right)^{2}$, em que $u$ representa a velocidade do êmbolo e $v_{x}$ representa a velocidade média das moléculas na componente paralela ao movimento do pistão. O sinal positivo aplica-se no caso de compressão; o sinal negativo, no caso de expansão. Anos depois, Carrera-Patiño ${ }^{20}$ detalhou um modelo cinético para predizer a evolução da temperatura de um gás ideal monoatômico em expansão/compressão adiabática com velocidade finita. Naturalmente o resultado de ambos os modelos é a coincidência do trabalho de expansão/compressão com o trabalho reversível se $u$ for igual a zero.

Há um impedimento para fazer $u$ tender a zero no Experimento de Clement-Desormes: a lentidão favorece a troca de calor. O processo reversível deve ser lento; o processo adiabático, rápido. A solução deste paradoxo concretiza-se: na diferença de pressão dentro e fora do recipiente; no diâmetro do gargalo; no tempo de abertura da rolha; na espessura da parede. Os dois primeiros definem a velocidade do jato de gás; os dois últimos, a velocidade de troca de calor. $\mathrm{O}$ 
dimensionamento é um compromisso entre os extremos. Consiste em fazer a velocidade do jato de gás baixa comparada à velocidade média das moléculas (o que aproxima o processo 1 da condição de reversibilidade, embora não a atinja) e rápida comparada à velocidade de troca de calor (o que aproxima o processo 1 de ser adiabático, embora também não alcance esta condição).

\section{SUPORTE EXPERIMENTAL}

\section{Parâmetros experimentais}

O dimensionamento descrito no parágrafo anterior encontra-se em Garland, Nibler e Shoemaker. A rolha é removida a cerca de 5 a $7 \mathrm{~cm}$ de distância no menor espaço de tempo possível. Valores típicos nas aulas experimentais do Instituto de Química na Universidade Federal de Uberlândia são $T_{a m b}=26,0^{\circ} \mathrm{C}, P_{1}=86,0 \mathrm{cmH}_{2} \mathrm{O}(101419 \mathrm{~Pa}$ com $g$ $\left.=9,79 \mathrm{~m} \mathrm{~s}^{-2}\right), P_{\text {atm }}=93000 \mathrm{~Pa}\left(\right.$ Oregon ${ }^{\circledR}$, modelo EB833), $P_{3}=22,0 \mathrm{~cm}$ $\mathrm{H}_{2} \mathrm{O}(95154 \mathrm{~Pa})$, e $t_{a b}$ entre 1 e $2 \mathrm{~s}$. Obtém-se com a Equação $1, \gamma_{\mathrm{ar}}=$ 1,36 . O erro é de quase $-3 \%$ em relação ao valor esperado $(\gamma=1,40)$ de um gás diatômico $\left(\mathrm{N}_{2}\right.$ e $\mathrm{O}_{2}$, do ar atmosférico).

\section{Incerteza dos resultados}

Magarvey, Bishop e Blackford ${ }^{18}$ investigaram o efeito do tempo de abertura, $t_{a b}$, e do diâmetro do gargalo sobre $\gamma$. Quanto mais rápida for a abertura, ou quanto mais estreito for o gargalo, maior é o valor obtido de $\gamma$. O valor esperado de um gás diatômico foi obtido em $t_{a b}$ $=0,90 \mathrm{~s}$ (diâmetro, $\phi, 0,63 \mathrm{~cm})$ e 1,60 s $(\phi=0,16 \mathrm{~cm})$. Estes valores foram usados para calcular o coeficiente $\left(\frac{\partial \gamma}{\partial t_{a b}}\right)_{\phi}$ a partir da Figura 1 destes autores. ${ }^{18}$ Os coeficientes em ambas as condições foram praticamente iguais, $-0,12 \mathrm{~s}^{-1}$. Assim, o erro relativo de $\gamma$ pode ser estimado em -0,12/1,40 (-8,6\%).

Decidir se os resultados experimentais ajustam-se melhor à Equação 1 ou à 2 exige incerteza dos resultados inferior a $2 \%$ em módulo (vide supra). Portanto, isto não é possível com o experimento de Clement-Desormes, mesmo com dimensionamento otimizado.

\section{Politropia}

O processo 1, idealmente, é adiabático. Entretanto, experimentalmente, há uma indesejada troca de calor por causa da imperfeição da parede do recipiente. A troca de calor neste tipo de situação caracteriza a politropia (ver definição rigorosa no Apêndice). A velocidade da troca de calor depende da condutividade térmica, $\kappa$. Então, quanto maior for a condutividade térmica do gás, maior deve ser sua influência no Experimento de Clement-Desormes.

Esta hipótese foi verificada. Os resultados do experimento de Clement-Desormes $\left(\gamma_{\mathrm{He}}=1,50 ; \gamma_{\mathrm{Ar}}=1,63\right)$ são menores que os resultantes de experimento independente ${ }^{2}$ (1,67 com ambos os gases). $\mathrm{O}$ erro em $\gamma_{\mathrm{He}}$ é muito maior em módulo $(-10,2 \%)$ do que o erro em $\gamma_{\mathrm{Ar}}$ $(-0,6 \%)$. De fato, o maior erro em módulo obtém-se com hélio, cuja condutividade térmica é quase nove vezes maior que a do argônio (156,7 e 17,9 $\mathrm{mW} \mathrm{m}^{-1} \mathrm{~K}^{-1}$, respectivamente). ${ }^{21}$ Assim, o resultado sistematicamente negativo origina-se na politropia. Adiante se demonstra este comportamento com base numa descrição macroscópica.

\section{Comentários finais}

Como não se pode alterar a condutividade térmica de um gás, não se pode dimensionar o equipamento com um gás e usá-lo com outro cuja condutividade térmica seja muito diferente sem aumentar o erro do resultado.
A politropia origina-se na diferença entre as temperaturas do sistema e da vizinhança e na incapacidade da parede do sistema barrar a transferência de calor. Esta situação caracteriza um processo de não equilíbrio. Troca de calor por gradiente de temperatura gera entropia. Geração de entropia é o único critério geral de irreversibilidade..$^{22}$ Por consequência, o processo de expansão é irreversível.

\section{CONCLUSÃO}

A expansão no processo 1 do experimento de Clement-Desormes é irreversível.

É possível ajustar o experimento de modo que duas condições antagônicas, necessárias para realização de processos reversíveis e adiabáticos, sejam satisfeitas simultaneamente: a velocidade do jato de gás na etapa 1 deve ser muito menor em comparação com a velocidade média das moléculas do gás; a etapa 1 deve ser rápida em comparação com a velocidade de troca de calor entre o recipiente e o exterior. As condições experimentais para realização do experimento de Clement-Desormes, descritas neste trabalho e na literatura citada, satisfazem a estas condições e são, em princípio, determináveis com base em modelo cinético-molecular. O uso da Equação 1 é aceitável dentro do erro experimental. O processo 1 pode ser tratado como sendo uma idealização ou como um caso limite, descrito pela reversibilidade.

A proximidade dos resultados obtidos com as Equações 1 e 2 não justifica nem a suposição de reversibilidade nem de irreversibilidade. É reflexo do ajuste descrito no parágrafo anterior.

O experimento de Clement-Desormes não é confiável na medição precisa de $\gamma$. Os resultados dependem de detalhes experimentais que podem variar com o gás usado. Se o interesse for exclusivamente o resultado, é preferível usar outro método. Se o interesse for o ensino de Físico-química, tem-se então fonte de numerosos detalhes conceituais, todos contextualizados, e fonte rica de discussão.

\section{AGRADECIMENTO}

Aos assessores, pelas correções e sugestões.

\section{APÊNDICE: POLITROPIA}

Considere-se um gás ideal (ou mistura de gases ideais sem reação química) em um sistema fechado e homogêneo, na ausência de campos. O primeiro princípio da Termodinâmica impõe: $d U=d q+d w$. A evolução temporal destas grandezas ao longo de um processo é: $\frac{d U}{d t}=\frac{d q}{d t}+\frac{d w}{d t}$, em que $t$ é o tempo ao longo do processo. Parametrizando: $\frac{d U}{d t} \frac{d t}{d T}=\frac{d q}{d t} \frac{d t}{d T}+\frac{d w}{d t} \frac{d t}{d T}$,obtém-se $\frac{d U}{d T}=\frac{d q}{d T}+\frac{d w}{d T}$. Distinguem-se três casos: ${ }^{23}(1) d q / d T=0$; (2) $d q / d T$ igual a infinito; e $d q / d T$ (3) igual a qualquer valor finito, não nulo. $\mathrm{O}$ primeiro caso define o processo adiabático; decorre do perfeito isolamento térmico entre o sistema e a vizinhança. É válida a equação $P_{1} V_{1}^{\gamma}=P_{2} V_{2}^{\gamma}$, se o processo for reversível. O segundo caso define o processo isotérmico e ocorre quando a energia interna é constante ao longo do processo (o que equivale a dizer que $U$ é temporalmente constante). É válida a equação $P_{1} V_{1}=P_{2} V_{2}$ (se a temperatura exterior for constante), porque se impõe a idealização de que o equilíbrio térmico se estabeleça instantaneamente. Finalmente, o terceiro caso define o processo politrópico. A politropia ocorre em todo processo real, porque a parede do sistema real nem obstrui totalmente a passagem de calor, nem permite a sua troca instantânea.

A relação entre pressão e volume de gases ideais nos processos politrópicos dá-se pela Equação A1. Desta forma, os três casos acima 
podem ser também caracterizados pelo expoente $k$ : (1) Se $k=\lambda$, o processo é adiabático; (2) Se $k=1$, o processo é isotérmico; e (3) Se $1<k<\gamma$, o processo é politrópico.

$$
P_{1} V_{1}^{k}=P_{2} V_{2}^{k}, 1<k<\gamma
$$

A Equação A1 pode ser obtida a partir do primeiro princípio da Termodinâmica. Os subscritos 1 e 2 deste apêndice não estão necessariamente relacionados aos estados 1 e 2 da Tabela 1 . Tratando-se de aplicação a um gás ideal, pode-se escrever $\frac{C_{V, m}}{T} d T=\frac{d q}{n T}-\frac{R}{V} d V$ No processo politrópico, o primeiro termo do segundo membro não pode ser ignorado. Por integração, $C_{V, m} \ln \frac{T_{2}}{T_{1}}=I_{q}-R \ln \frac{V_{2}}{V_{1}}$, com $I_{q}=\int[1 /(n T)] d q$. O termo $I_{q}$ não tem significado físico especial, exceto no caso reversível, em que $I_{q}=\Delta S_{m}$, isto é, a variação de entropia molar do gás. Eliminando a temperatura, obtém-se $\ln \left(\frac{P_{2} V_{2}^{\gamma}}{P_{1} V_{1}^{\gamma}}\right)=\frac{I_{q}}{C_{V, m}}$. Rearranjando: $\frac{P_{2} V_{2}^{\gamma-I_{q} /\left[V_{\gamma, m} \ln \left(V_{2} / V_{1}\right)\right]}}{P_{1} V_{1}^{\gamma-I_{q} /\left[C_{\gamma, m} \ln \left(V_{2} / V_{1}\right)\right]}=1}$. Obtém-se a Equação A1, definindo $k$ por:

$$
k=\gamma-\frac{I_{q}}{C_{V, m} \ln \left(V_{2} / V_{1}\right)}
$$

Considere-se agora o gás inicialmente em equilíbrio térmico com a vizinhança. Na expansão, o volume aumenta e $V_{2} / V_{1}>0$; o gás realiza trabalho à custa da diminuição da energia interna e, por isso, resfria-se; a parede permite certa troca de calor, da vizinhança para o sistema, de modo que $I_{q}$ é positivo. Portanto, $k>\gamma$. Na compressão, o volume diminui e In $V_{2} / V_{1}>0$; o gás aquece-se, calor sai e $I_{q}$ é negativo. Igualmente, $k>\gamma$, ficando demonstrada a aplicação desta desigualdade a qualquer processo politrótico, inicialmente em equilíbrio térmico com sua vizinhança.

Há uma particularidade: $k$ não é uma constante porque depende do volume. Para ilustrar, considere-se a aplicação da Equação A1 a três estados: $P_{1} V_{1}{ }^{k}=P_{2} V_{2}{ }^{k}$ e $P_{1} V_{1}^{k^{\prime}}=P_{3} V_{3}{ }^{\prime}$. Entretanto, $k^{\prime} \neq k$, diferentemente dos processos adiabáticos e reversíveis, em que vale $P_{1} V_{1}^{\gamma}=P_{2} V_{2}^{\gamma}=P_{3} V_{3}^{\gamma}$.

De forma geral, é possível que $\ln P$ varie linearmente com $\ln V$. $\mathrm{O}$ coeficiente angular, $k$, pode assumir qualquer valor, dependendo do sistema. Em textos direcionados para aplicação instrumental, ${ }^{24}$ é comum considerar o coeficiente angular constante, sem mencionar a dependência com o volume.

\section{REFERENCIAS E NOTAS}

1. Desormes, C. B.; Clement, N.; J. de Physique Hist. Nat. 1819, 89, 428.

2. Moore, W. M.; J. Chem. Educ. 1984, 61, 1119.

3. Meyer, E. F.; Stewart, G. H.; J. Chem. Educ. 1988, 65, 282.

4. Garland, C. W.; Nibler, J. W.; Shoemaker, D. P.; Experiments in Physical Chemistry, $7^{\text {th }}$ ed., McGraw Hill: Boston, 1996, p. 107-110.

5. Holden, G. L.; J. Chem. Educ. 2007, 84, 513.

6. Bertrand, G. L.; McDonald, H. O.; J. Chem. Educ. 1986, 63, 252.

7. Hornack, F. M.; J. Chem. Educ. 1996, 73, 965.

8. Levine, I.; Physical Chemistry, McGraw Hill Internat. Book Co.: São Paulo, 1979, p. 114.

9. Buep, A. H.; Czekalski, M; Baron, M.; J. Chem. Educ. 1988, 65, 416.

10. Bertrand, G. L.; J. Chem. Educ. 2005, 82, 874.

11. J. A. Beattie definiu trabalho como qualquer quantidade que flui através da fronteira do sistema durante a alteração no seu estado e é completamente conversível na elevação de um peso na vizinhança. Ver ref. 15.

12. Haase, R.; Themodynamics of Irreversible Processes, $1^{\text {st }}$ ed. ampliada, Dover Pub. Inc.: Nova Iorque, 1990.

13. Fermi, E.; Thermodynamics, Dover Publications Inc.: Nova Iorque, 1936, p. 13.

14. Prigogine, I.; Defay, R.; Thermodynamique Chimique, Editions Desoer: Liege, 1950.

15. Castellan, G.; Fundamentos de Físico-química, Livros Técnicos e Científicos Editora S.A.: São Paulo, 1986, p. 116.

16. Sears, P. W.; An Introduction to Thermodynamics, the Theory of Gases and Statistical Mechanics, Addison-Wesley Pub. Inc.: Londres, 1966, p. 3.

17. Nery A. R. L.; Bassi ,A. B. M. S.; Quim. Nova 2009, 32, 522; Moreira, N. H.; Bassi, A. B. M. S.; Quim. Nova 2001, 24, 563; http://chemkeys. com/en/wp-content/themes/chemkeysen/articleI.php?u=bWF0ZW1hdG ljYS11LXR1cm1vZGluYW1pY2E=, acessada em Junho 2010.

18. Magarvey, R. H.; Bishop, R. L.; Blackford, B. L.; Am. J. Phys. 1961, 29 , 274.

19. Bauman, R. P.; J. Chem. Educ. 1964, 41, 102; Chesik, J. P.; J. Chem. Educ. 1964, 41, 674; Kokes, R. J.; J. Chem. Educ. 1964, 41, 675.

20. Carrera-Patiño, M. E.; J. Chem. Phys. 1988, 89, 2271.

21. Lide, D. R., ed.; CRC Handbook of Chemistry and Physics, 86a ed., Taylor \& Francis Group, LLC: Nova Iorque, 2005.

22. Prigogine, I.; Thermodynamics of Irreversible Processes, Interscience Publishers (Wiley): Nova Iorque, 1961, p. 17.

23. Macedo, H.; Dicionário de Física, Ed. Nova Fronteira S.A., Rio de Janeiro, 1976.

24. Borgnakke, C.; Sonntag, R. E.; Fundamentos de Termodinâmica, Ed. Blucher: São Paulo, 2009, p. 71. 